\title{
Octave Spanning Mid-Infrared Frequency Comb Generation in Silicon Nanophotonic Wire Waveguides
}

\author{
Bart Kuyken ${ }^{1,2}$, Takuro Ideguchi ${ }^{3}$, Simon Holzner ${ }^{3}$, Ming Yan $^{3}$, Theodor W. Hänsch ${ }^{3}$, Joris Van \\ Campenhout $^{4}$, Peter Verheyen ${ }^{4}$, Roel Baets ${ }^{1,2}$, Gunther Roelkens ${ }^{1,2}$, Nathalie Picqué ${ }^{3}$ \\ ${ }^{I}$ Photonics Research Group, Department of Information Technology, Ghent University-imec, Ghent, Belgium \\ ${ }^{2}$ Center for Nano- and Biophotonics (NB-Photonics), Ghent University, Ghent, Belgium \\ ${ }^{3}$ Max Planck Institut für Quantenoptik, Garching Germany \\ ${ }^{4}$ imec, Kapeldreef 75, Leuven, Belgium \\ Email: Bart.Kuyken@intec.ugent.be
}

\begin{abstract}
A mid-infrared octave spanning frequency comb is generated in a silicon waveguide. By beating the generated comb on a photodetector with a narrow linewidth lightsource the linewidth of the lines is measured to be $<100 \mathrm{kHz}$. (C) 2014 Optical Society of America

OCIS codes: (190.4380) Nonlinear optics, four-wave mixing; (190.4390) Nonlinear optics, integrated optics
\end{abstract}

\section{Introduction}

The mid-infrared spectral region is a domain of interest to many areas of science and technology [1] including molecular fingerprinting, trace gas sensing, thermal imaging, and biomedical diagnostics. Laser frequency combs, which emit a broad spectrum consisting of discrete, evenly spaced narrow lines, have the potential to significantly expand the performance of precision spectroscopy and broadband molecular sensing in this region. Extension of frequency comb techniques to the mid-infrared spectral region is thus currently under exploration [2]. The recent significant progress $[3,4]$ in developing mid-infrared photonics platforms in $\mathrm{Si}$, which is highly transparent in the mid-infrared, offers exhilarating perspectives for versatile frequency comb generation, due to CMOS compatibility and the ease of fabrication. Here we demonstrate an octave spanning mid-infrared frequency comb through supercontinuum generation in silicon wire waveguides. We verify the phase coherence of the comb generated in a photonic wire waveguide by monitoring free-running beat notes between the supercontinuum and narrow linewidth lasers.

\section{Experimental results}

The $1 \mathrm{~cm}$-long silicon nanophotonic wire used in the experiment is fabricated in imec's CMOS pilot line, on a 200 $\mathrm{mm}$ silicon-on-insulator (SOI) wafer consisting of a $390 \mathrm{~nm}$ silicon waveguide layer on a $2 \mu \mathrm{m}$ buried oxide with no top cladding. The dimensions of the photonic wire are shown in the inset of Figure 1 a). The waveguide is slightly over etched by $10 \mathrm{~nm}$. Using a cutback method, the propagation loss in the $2200-2400 \mathrm{~nm}$ band is estimated to be < $0.2 \mathrm{~dB} / \mathrm{cm}$.

For the experiment the quasi TE mode of the waveguide is excited by a home-made femtosecond optical parametric oscillator (OPO) with a repetition frequency of $100 \mathrm{MHz}$. When the center wavelength of the output pulses of the optical parametric oscillator is tuned to a center wavelength of $2290 \mathrm{~nm}$, about 70-fs long pulses are produced. The average output power coming out of the OPO is $25 \mathrm{~mW}$. The pulses are coupled to the chip using a high numerical aperture (NA) chalcogenide lens. The coupling loss to the chip is $12 \mathrm{~dB}$ resulting in an on-chip peak power of $264 \mathrm{~W}$. The output of the photonic chip is coupled via a high NA lens to a Fourier transform infrared spectrometer (FTIR). The output spectrum of the chip recorded with the FTIR is shown in Figure $1 \mathrm{~b}$ ). The spectrum of the input pulses is also shown in the figure in red. As can be seen in Figure $1 \mathrm{~b}$ ), the supercontinuum generated in the $1 \mathrm{~cm}$ silicon photonic wire spans from $1.55 \mu \mathrm{m}$ up to $3.3 \mu \mathrm{m}$. The broadening is achieved by pumping the silicon wire waveguide in the anomalous dispersion region, close to the zero dispersion wavelength of the photonic wire waveguide as shown in Figure $1 \mathrm{~b}$ ). 

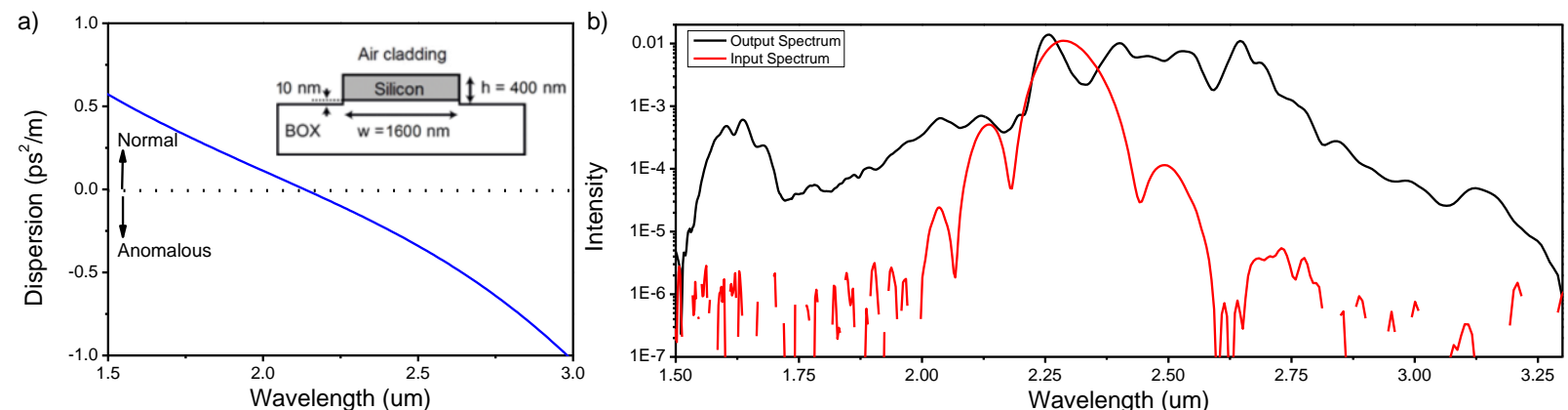

Figure 1: a) The second order dispersion of the photonic wire waveguide. The inset in figure a) shows the geometry of the silicon photonic wire used. The black curve in Figure $1 \mathrm{~b}$ ) shows the output spectrum of the silicon photonic wire waveguides, an octave spanning supercontinuum from $1.55 \mu \mathrm{m}$ to $3.3 \mu \mathrm{m}$ is generated. The input and output spectra are in arbitrary units.

\section{Phase coherence of the generated supercontinuum}

Spectral broadening in a highly nonlinear waveguide can introduce noise causing a loss of coherence in the spectrum. We thus characterize the phase coherence of the spectrum at the output of the chip by measuring freerunning beat notes between the output of the chip and narrow line-width continuous wave lasers at different frequencies across the octave spanning spectrum. First the supercontinuum beats with a continuous wave (cw) narrow linewidth fiber laser at $1586 \mathrm{~nm}$ (Koheras Adjustics E15, line-width 1 kHz at $120 \mu \mathrm{s}$ ) on a fast InGaAs photodetector. A portion of the resulting radiofrequency (RF) spectrum of the output of the photodetector is shown in Figure 2 a). A resolution bandwidth of $10 \mathrm{kHz}$ is chosen to limit the spread in beat-note frequency due to variation in the unlocked optical sources. The same experiment was performed in the mid-infrared and we present the beat note measurement of the supercontinuum and a mid-infrared $\mathrm{cw}$ tunable optical parametric oscillator (Argos, Aculight, line-width $\sim 60 \mathrm{kHz}$ at $500 \mu \mathrm{s}$ ) when the cw OPO is tuned to a wavelength of 2418 (Fig.2b) and $2580 \mathrm{~nm}$ (Fig. 2c) respectively. For these two measurements, a 100-MHz InGaAsSb photodiode (D124, Redwave Labs) is used. In all these cases, the narrow line-width beat note shows that the generated supercontinuum is coherent. All beat notes exhibit about the same line-width of $50-70 \mathrm{kHz}$ and no broadening of the beat signals involving the comb modes at the chip output is observed relative to the femtosecond OPO pump (not shown in the figure). The results demonstrate good phase coherence of the broadened spectrum generated by the Si chip.
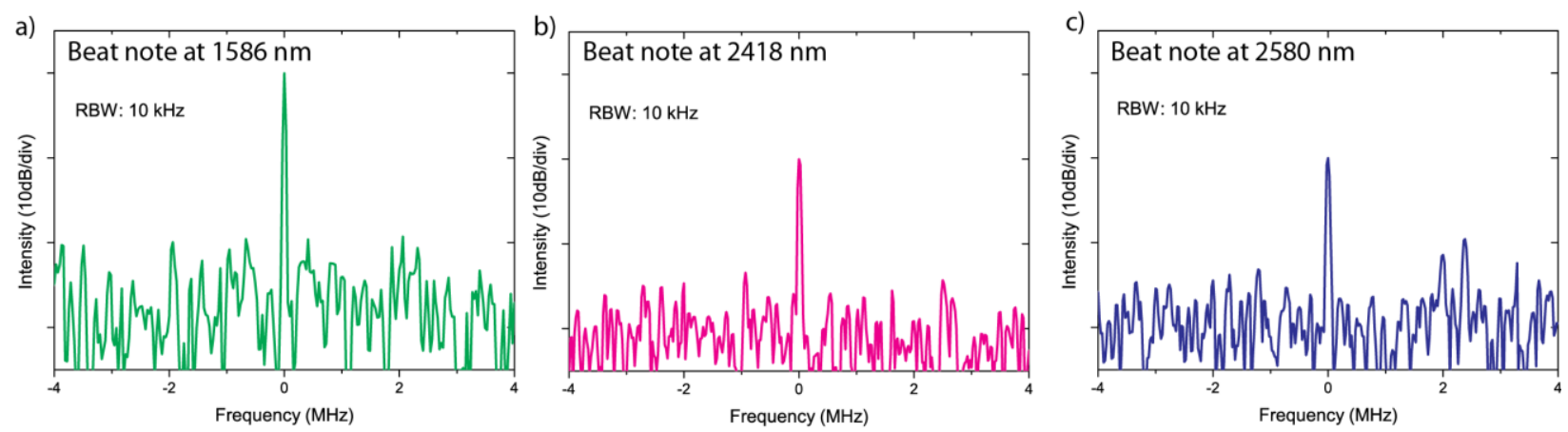

Figure 2: The RF spectrum of a photodetector measuring the beat note of the supercontinuum comb sand a cw narrow linewidth laser at a) 1586 $\mathrm{nm}, \mathrm{b}) 2418 \mathrm{~nm}$, c) $2580 \mathrm{~nm}$. No other lines are observed in the measurement with a frequency span equal to half the repetition frequency. All free-running beat notes are measured with a resolution bandwidth (RBW) of $10 \mathrm{kHz}$.

\section{Acknowledgements}

This work was supported by the FP7-ERC-MIRACLE, FP7-ERC-InSpectra and FP7-ERC-Multicomb projects. Bart Kuyken acknowledges a scholarship provided by the special research fund of Ghent University

\section{References}

[1] J. G. Crowder, et al., "Infrared methods for gas detection," in Mid-Infrared Semiconductor Optoelectronics. New York: Springer-Verlag, 2006.

[2] A. Schliesser, et. al. "Mid infrared frequency combs", Nature Photonics 6, 440 (2012).

[3] M. Milosevic, et al., "Silicon waveguides and devices for the mid-infrared," Applied Physics Letters 101, 121105 (2012).

[4] B. Kuyken, et al., "Mid-infrared to telecom-band supercontinuum generation in highly nonlinear silicon-on-insulator wire waveguides," Optics Express 19, 20172 (2011). 